\title{
Esbozo de una nueva política forestal peruana
}

\author{
Outline of a new Peruvian forest policy
}

Marc J. Dourojeanni ${ }^{1, *}$

Recibido: 20 abril 2019 | Aceptado: 11 junio 2019 | Publicado en línea: 30 junio 2019 Citación: Dourojeanni, MJ. 2019. Esbozo de una nueva política forestal peruana. Revista Forestal del Perú 34(1): 4-20. DOI: http://dx.doi.org/10.21704/rfp.v34i1.1244

\begin{abstract}
Resumen
Ninguna de las políticas forestales adoptadas en el Perú durante el último medio siglo ha dado los resultados esperados. Una de las causas es que la política ha sido usualmente una mera formalidad, siempre precedida por la legislación forestal, estando ambas desconectadas. Pero, la causa principal sería la persistencia en la estrategia de transferir la responsabilidad del manejo forestal de los bosques naturales al sector privado, a pesar de las evidencias de que esos bosques tienen más valor presente y futuro por los servicios ambientales que prestan que como productores de madera. Se propone que, en el futuro, el manejo de los bosques naturales sea ejercido directamente por el Estado, dando prioridad clara a los servicios ambientales. La extracción de la madera, si es justificada, continuaría siendo responsabilidad del sector privado, mediante concursos públicos o licitaciones bajo estrecha supervisión social. Varias otras medidas complementarias son propuestas, entre ellas: valorización de la madera de bosques naturales, incentivos a la forestación, reforestación y manejo de bosques secundarios, el pago efectivo por servicios ambientales del bosque natural, cambio en el enfoque sobre bosques de protección, revisión del concepto de clasificación de tierras por capacidad de uso mayor, creación de incentivos efectivos al manejo forestal en tierras indígenas. También se proponen arreglos administrativos para conducir la nueva política, encargándola al Ministerio del Ambiente y a las regiones; mientras que, lo relativo a las plantaciones forestales productivas debe ser promovido por el sector agrario.
\end{abstract}

Palabras clave: Perú, política forestal, legislación forestal, concesiones, manejo forestal, forestación y reforestación, uso de la tierra

\footnotetext{
${ }^{1}$ Facultad de Ciencias Forestales, Universidad Nacional Agraria La Molina (UNALM), Av. La Molina s/n, La Molina, Lima, Perú.

* Autor de Correspondencia: marc.dourojeanni@gmail.com
} 


\begin{abstract}
None of the forest policies adopted in Peru during the past half century has given the expected results. The policy has often been a mere formality, always preceded by forest legislation, both of which are disconnected. But, the main cause of failure is the persistence in the strategy of transferring natural forest management responsibility to the private sector, despite the evidence that these forests have more present and future value as environmental services than as timber producers. It is proposed that, in the future, the management of natural forests be directly exercised by the State, giving clear priority to environmental services. Logging, if justified, would continue to be the responsibility of the private sector, through public tenders or bids under close social supervision. Several other complementary measures are proposed, including valuation of wood from natural forests, incentives for afforestation, reforestation and management of secondary forests, effective payment for environmental services of standing natural forest, change in the focus on protection forests, review of the current concept of land use classification, provision of effective incentives for forest management on indigenous lands. Administrative arrangements are also proposed to conduct the new policy, entrusting it to the Ministry of the Environment and to the regions, while most matters related to forest plantations must remain in the Agrarian Sector.
\end{abstract}

Key words: Peru, forest policy, forest legislation, forest concessions, forest management, forestation and afforestation, land use

\section{Introducción}

El Perú moderno tiene una política forestal desde la década de 1940, cuando el gobierno asumió formalmente la gestión de los bosques a través del recién creado Ministerio de Agricultura. Pero, como es obvio, hubo alguna forma de política forestal desde que existieron pueblos organizados en el territorio que hoy es el Perú. A partir de 1945, con la creación de la Dirección de Colonización y Bosques, fueron preparadas y aprobadas varias políticas forestales, algunas escuetas y otras amplias, casi siempre acompañadas de planes de desarrollo forestal. Cabe destacar las que fueron diseñadas bajo el régimen del Servicio Forestal y de Caza entre 1961 y 1969. Desde entonces, cada nuevo gobierno, y a veces cada nuevo jefe del sector forestal, dispuso la preparación de una política forestal nacional o, por lo menos, reajustes en la previamente existente. La que está vigente fue aprobada en 2013, por Decreto Supremo No 009-2013-MINAGRI (SERFOR 2013).

En este análisis, en el que no se aborda el tema de la fauna silvestre, se pretende proponer alternativas para una política forestal na- cional sustancialmente diferente de la actual, y de las anteriores que, como todo indica, no han funcionado.

\section{La situación}

A pesar de que hay una política forestal nacional oficial, la impresión de los que examinan el desempeño del sector es que ésta no existe, que es inadecuada o, en todo caso, que no se aplica. La Defensoría del Pueblo (2010) concluye, que a pesar de que desde el año 2000 las políticas forestales se han orientado a lograr una mayor sostenibilidad en el manejo de los recursos forestales, este objetivo no se ha alcanzado. Suárez (2012) sostiene que la gestión forestal peruana falla en las tres dimensiones del desarrollo sostenible: no es sostenible ecológicamente, no es inclusiva socialmente, no es competitiva económicamente. A la misma o similar conclusión llegaron, entre muchos otros, Pautrat y Lucich (2006) y Dourojeanni et al. (2016).

De hecho, no es necesario apelar a diagnósticos complejos para conocer la dimensión del fracaso de la política forestal, incluyendo sus leyes asociadas. En efecto, todos los indicadores 
de resultados de cualquier política forestal usada hasta la actualidad evidencian que éstas en el Perú no funcionaron. Así, la deforestación nunca dejó de crecer y a pesar de diversas estrategias realizadas (Dourojeanni 2011, 2013c) se revela enorme (MAAP 2017, 2018a, 2018b); el aporte de la producción de madera y otros productos forestales al PBI nacional continúa siendo muy bajo pese al enorme potencial forestal nacional, siendo el Perú un importador neto de esos bienes (INEI 2017, SERFOR 2017b); no existe manejo sostenible del bosque productor ni siquiera en las concesiones formales (Dourojeanni 2009, Finer et al. 2014, Sierra 2018a); la corrupción en el sector continúa sin cambios a pesar de los esfuerzos (IEA 2012; Sierra 2018b, 2019; Global Witness 2019); los desastres naturales que podrían ser prevenidos por bosques protectores aumentan año a año (Dourojeanni 2017, MINAM 2015, INEI 2018); los pueblos indígenas y los pobladores tradicionales no se benefician del recurso forestal (Chirif 2018, Sierra 2019a); los beneficios prometidos en recompensa por servicios ambientales para frenar el cambio climático, pese a los esfuerzos del Ministerio del Ambiente, siguen siendo más promesas que realidades (Espinoza y Feather 2011, Egúsquiza 2012); la degradación de los bosques naturales, por descremado y por la falsa agroforestería de cacao y café, continúa aceleradamente y sin control (Sierra 2019b); y, para terminar, la tan prometida reforestación, cuyas estadísticas oficiales son abiertamente fantasiosas, avanza a paso muy lento y continúa enfrentando grandes dificultades (SERFOR 2017b).

A lo largo del último medio siglo hubo varios éxitos destacables, más bien experimentos, inclusive en manejo de bosques tropicales, reforestación andina y selvática, desarrollo comunitario basado en productos del bosque, en la investigación y capacitación técnica e, inclusive, en gestión forestal (Dourojeanni 1990, 2009). Pero mencionarlos en un contexto de análisis general es fuera de lugar pues fueron parciales, circunscritos en el espacio o muy limitados en el tiempo, no consolidando éxitos ni aprovechando las experiencias. Como siem- pre, hay una que otra excepción, como es el caso de la reforestación de Cajamarca, que destaca por su amplitud, duración y solidez social (Dourojeanni 2009, Carton y Chávez 2018).

Es decir, que la evidencia de que las políticas forestales aplicadas en el Perú no sirvieron ni sirven es indiscutiblemente abrumadora. La única parte de la política forestal que ha sido razonablemente cumplida desde la década de 1960 es la que se refiere a la conservación de muestras representativas de los ecosistemas naturales y de la diversidad biológica contenida en ellas que, desde 2008, es conducida por el Servicio Nacional de Áreas Naturales Protegidas por el Estado (SERNANP) entidad adscrita al Ministerio del Ambiente.

\section{Algunos elementos que explican el fraca- so de las políticas forestales peruanas}

Es probable que la causa principal de la falta de aplicación de una política forestal sea, simplemente, que esté mal hecha y que efectivamente no sirva para otra cosa que cumplir una formalidad burocrática. La lectura de la política forestal vigente (SERFOR 2017a) confirma eso. A primera vista no dice nada errado y todo lo que plantea es deseable. Pero, en una segunda lectura, se observa que no contiene ninguna propuesta concreta ni estrategia para asegurar que cada propósito enunciado sea realizable. En realidad, se trata de una colección de buenos propósitos aderezados con frases políticamente correctas. Además, exceptuando declaraciones forzadas por las nuevas realidades como el cambio climático, el papel político creciente de los indígenas amazónicos y otros temas de moda como género, transparencia y participación, en lo esencial, las políticas forestales peruanas no han cambiado en 60 años.

Es decir, que la política continúa girando en torno a la producción de madera en base a concesiones -antes llamadas contratos- y a la falta de decisión sobre qué hacer con los pequeños extractores. La esencia de la política forestal se carga al sector privado, con la demostradamente vana esperanza de que éste cumpla con 
realizar el manejo sostenible de los bosques y se engaña al no enfrentar directamente la informalidad de los pequeños extractores, habiéndose inventado en diferentes épocas falsas soluciones como los "contratos de hasta 1000 ha" (Ley N ${ }^{\circ} 21147$ ) o las "concesiones con fines de forestación y reforestación” (Ley N ${ }^{\circ} 27308$ ), entre otras. Llama pues la atención de que a pesar de que nada ha funcionado hasta el presente, se persista en la misma política, sin cambios sustantivos.

Parte del problema es que la última política forestal, como otras previas, fueron hechas después de aprobar una nueva legislación forestal. De hecho, la verdadera política forestal peruana vigente se desprende de la Ley $\mathrm{N}^{\circ}$ 29763 del año 2011, que fue muchísimo más discutida que la política, que, además, fue aprobada dos años después (Galarza y La Serna 2005; Pautrat et al. 2010; Dourojeanni 2010, 2013a, 2013b; Salo et al. 2013) continuando hasta el presente día (Che Piu 2018). Es decir, que, tanto en el Perú como en otros países, la legislación es la que determina la política y no al revés, como debería ser, si es que se quiere viabilizar la ejecución de la política. La FAO (2010a, 201b) recuerda correctamente que el propósito primordial de una legislación es la distribución y el cumplimiento de los derechos y responsabilidades relacionados con los bosques, y no el establecimiento de una visión, meta o estrategia. Por eso, la política -es decir un acuerdo sobre una dirección estratégicadebe ser formulada antes de que cualquiera de sus aspectos pueda ser jurídicamente vinculante. De otra parte, como bien se sabe, la legislación forestal tampoco es cumplida (Arce y Dourojeanni 1990, Dourojeanni 2010) ni cumplible (Dourojeanni 2013b).

Además, existen muchos otros factores que contribuyen a la inoperancia de las políticas forestales en el Perú. Algunos son intrínsecos al sector forestal, como el enorme tamaño, la dispersión y la complejidad extrema del recurso, junto a la marcada debilidad institucional. Otros problemas nacionales como la pobreza e informalidad, la corrupción generalizada, el bajo nivel educativo y la falta de prioridad política para temas ambientales, legislaciones y planeamientos sectoriales incoherentes $y$, en general, la desorganización e ineficiencia del Estado, entre otros males nacionales, que son más acentuados precisamente en las regiones boscosas del país, inciden directamente sobre la viabilidad de cualquier política forestal, aunque en principio, esos mismos factores deberían ser llevados en cuenta al diseñarla.

\section{Sugerencias para una verdadera política forestal}

Como hemos visto, una política forestal que sirva depende obviamente de la posibilidad de ser implementada mediante un instrumento legal con soluciones viables, aceptables social, económica y ambientalmente. Debe fundamentarse en un diagnóstico crudo y absolutamente honesto de la situación, lo cual es muy raro. Los diagnósticos del sector forestal son, tradicionalmente, grandes acumulaciones de informaciones en su mayoría bien conocidas o reiterativas que carecen de análisis. La principal cuestión que el diagnostico debe revelar es por qué la política anterior no ha dado resultados. Es sobre la base de esa respuesta que se debe iniciar el largo y complejo proceso de consulta a las partes involucradas directa e indirectamente, cuidando que la participación sea efectiva y equitativamente balanceada. La parte que representa al Estado no puede renunciar a hacer valer los principios fundamentales ambientales y sociales como sostenibilidad, conservación, servicios ambientales, inclusión, participación, ciencia, interculturalidad y género, los cuales deben de regir la nueva política forestal. El Estado, en esa discusión, representa los intereses de todos los peruanos y no única ni principalmente los de las partes directamente involucradas como madereros, industriales o indígenas.

Se supone que si el diagnóstico o, como en el caso que se analiza, las evidencias demuestran que las políticas previas no funcionaron, la discusión de la nueva política no puede limitarse a cambiar nombres y hacer retoques preciosistas o menciones a los temas de moda. Debe innovar, debe usar la imaginación y proponer 
nuevos rumbos. En pocas palabras, debe ser diferente. A continuación, se mencionan los temas que a juicio del autor son los principales a abordar y en cada caso, se proponen algunas alternativas de políticas a ejecutar.

\section{¿Cuál es la función principal del bosque nat- ural? ¿Brindar madera o servicios ambien- tales?}

Lo primero que debe decidir la política forestal es lo que quiere hacer con sus bosques naturales. Como mencionamos, hasta el día de hoy la decisión siempre ha sido explotarlos para producir madera y otros bienes. Todo lo demás, en las políticas forestales peruanas, ha sido secundario y subordinado a esa finalidad.

Sin embargo, cualquier análisis económico integral demuestra que el bosque natural tropical es mucho más valioso por sus servicios ambientales que como productor de madera y otros bienes (de Groot et al. 2012). Basta con mencionar los servicios ambientales, $y$ comparar el aporte a la economía peruana que brinda el turismo influenciado por las áreas naturales protegidas, con el de la producción de madera de los bosques naturales (León 2007, León et al. 2009, Glave y Pizarro 2002, Vilela et al. 2018). Para el futuro de cualquier nación es mucho más importante tener bosques naturales que explotarlos. La madera puede ser producida en bosques cultivados o reemplazada por otros materiales, como plástico y metales. Pero la calidad del aire, la disponibilidad de agua, la regulación del clima y el mantenimiento de la diversidad biológica, son insustituibles y, lo que es más importante, son esenciales para la vida (Prance y Lovejoy 1984, Myers 1984, Llerena y Yalle 2014). Esta realidad ha tomado mayor vigencia con las evidencias de la importancia del bosque tropical en relación con el ciclo del agua (Salati et al. 1979, Salati y Vose 1984) y como sumidero de carbono tanto en la biomasa como en el subsuelo y del impacto de su liberación con relación al cambio climático (McClain et al. 2001). La confirmación de la teoría de los "ríos voladores" cuya fuente es la transpiración de los bosques amazónicos, ha agregado otra dimensión a esas realidades
(Marengo et al. 2011, Makarieva et al. 2014, Nobre 2016). La valorización de esos servicios demuestra que su importancia, en términos económicos, potencialmente supera por innumerables veces al aporte económico de la madera de esos mismos bosques naturales (Glave y Pizarro 2002; Nobre 2016).

Es decir que la política forestal debe decidir si su prioridad para los bosques naturales seguirá siendo la producción de madera o si, en el futuro, la prioridad para esos bosques será su manejo para el mantenimiento y el mejoramiento de la provisión de servicios ambientales. Si ese fuera el caso, como la lógica de la información disponible conmina, se desprenden tres grandes acciones en la política forestal peruana. La primera es que se debe dar un impulso sin precedentes a la silvicultura -forestación, reforestación- con fines industriales en las tierras deforestadas en la Selva y en las tierras disponibles en la Costa y Sierra. La segunda es obtener una decisión nacional firme de poner límites a la expansión agropecuaria sobre bosques naturales, pues de nada serviría no explotar madera de los bosques si, en cambio, estos son eliminados para otros usos. Se debe discutir y coordinar con otros sectores de la sociedad, ese límite y, eventualmente, volver a discutir el tema de la "deforestación cero" (Brown y Zarin 2013) lanzado durante la gestión del ministro Antonio Brack (2010). Y, la tercera, es desarrollar seriamente los mecanismos de compensación económica para los segmentos de la sociedad que dejarán de explotar madera en sus bosques bien sea en términos de fijación de carbono y/o de regulación de flujo hídrico, entre otros servicios a valorizar.

La decisión de dar prioridad a los servicios ambientales de los bosques naturales no tiene necesariamente que ser radical y asimismo puede ser parcial, por lo menos en una primera etapa, para posibilitar la transición. En ese caso se debe estudiar y discutir la proporción y localización de los bosques de producción que continuarían siendo explotados y bajo qué modalidad. Asimismo, las alternativas prácticas para evitar la explotación -ilegal- de los 
bosques que no serán manejados para realizar servicios ambientales.

\section{El manejo sostenible de los bosques naturales es responsabilidad directa del Estado}

Si bien hasta el presente no existen bosques tropicales que sean manejados en forma indiscutiblemente sostenible (Dourojeanni 1999, Zimmerman y Kornos 2012) existen algunas evidencias de que es posible, aunque a un alto costo, hacer un manejo ecológicamente aceptable (Putz y Romero 2015). Lo que sí está bien demostrado por la práctica en el Perú es que hacer manejo más sostenible no es del interés del sector privado maderero, tanto por su complejidad técnica como por su costo elevado y porque los beneficios de un buen manejo, o sea los servicios ambientales, son principalmente para la sociedad en su conjunto. Es decir, que la responsabilidad del manejo de los bosques naturales públicos debe ser del Estado.

Es preciso recordar que el manejo forestal es la expresión final y la más decisiva de la gestión forestal (Akishin 2014). La gestión forestal, de acuerdo con la política vigente, es ejercida por el Estado. Pero como hemos explicado, eso no ocurre ya que el manejo forestal es enteramente relegado al sector privado. Los planes de manejo forestal que son preparados por las empresas para cada concesión están basados generalmente en inventarios forestales deficientes para reducir costos, y aunque eventualmente los planes sean de calidad razonable, raramente son aplicados y nunca lo son en forma consistente a lo largo del tiempo (Dourojeanni 2018c). Al respecto de los planes de manejo, es importante que la política forestal tome en cuenta que ha sido demostrado que las rotaciones tan cortas como las que en teoría se practican en el Perú, no garantizan la sustentabilidad del recurso (Zimmerman y Kornos 2012). Es decir, que los planes de manejo de la actualidad son apenas ritos para poder explotar, transportar y comerciar la madera. El resultado es que casi toda la madera extraída de los bosques naturales amazónicos al ser formalmente ilegal también es técnicamente ilegal, lo que significa que su extracción compromete la capacidad de esos bosques de continuar brindando madera y servicios ambientales (Dourojeanni 2018c).

Para que la gestión forestal sea seria, los planes de manejo deben ser preparados in situ por las agencias del gobierno competentes, como el antes mencionado SERFOR o por las administraciones forestales regionales debidamente instrumentadas. En lugar de las concesiones existentes habría unidades forestales permanentes con una adecuada localización, tamaño y otras características definidas por la autoridad forestal pertinente, como por ejemplo son los bosques nacionales en otros países. Debe quedar claro que no se sugiere que el Estado explote los bosques naturales. Su función es estudiarlos, inventariarlos, desarrollar los planes de manejo y supervisar su implantación y ejecución. Cada año, las administraciones forestales regionales, subastarían la madera que técnicamente puede ser extraída del tramo anual de corta de cada unidad forestal manejada, mientras que las empresas madereras enviarían su personal y maquinaria para extraer el lote de madera adquirido, bajo la supervisión del profesional forestal encargado por el Estado responsable de esa unidad. Con lo recaudado por la madera subastada, las administraciones forestales regionales pueden ejercer control, vigilancia y monitoreo; actualizar planes de manejo, contratar servicios de empresas silviculturales o de mantenimiento de caminos forestales. Es decir que el servicio forestal, incluidas las administraciones regionales, pasaría a manejar los bosques naturales y no existirían concesiones, ni necesidad de un organismo supervisor de estas. Sería una tremenda simplificación de la gestión forestal (Dourojeanni 2010, 2018a).

El plan de manejo de los bosques administrados por cada unidad forestal debe considerar abastecer, en la medida de lo posible, la demanda de las industrias localizadas en ese ámbito (Dourojeanni 2018c). Esos planes son, obviamente, discutidos y aprobados con la participación de todos los actores y su aplicación es supervisada por la sociedad local, reunida en comités o consejos forestales, con participación de las industrias madereras que aprovechan la 
madera de esos bosques. Ese es un buen mecanismo de control y supervisión ejercido por la propia sociedad, seguramente más eficiente que un organismo nacional externo como el OSINFOR.

La propuesta de que el manejo forestal sea realizado directamente por el Estado no reduce el rol del sector privado que continúa siendo el responsable de extraer la madera del bosque, transportarla, procesarla y comercializarla. Lo único que cambia es que la tan criticada y hasta ahora ineficiente estrategia de dar concesiones grandes -o pequeñas- sobre el recurso forestal es reemplazada por subastas periódicas por volumen y de especies de madera que pueden ser extraídas del bosque, de acuerdo con el plan de manejo de cada unidad (Dourojeanni 2018c). En el Perú prácticamente no existen bosques naturales privados por lo que la propuesta escuetamente descrita es perfectamente viable. Bosques de comunidades nativas o campesinas pueden, o no, adherirse a esa forma de manejo.

Esa es la forma en que se manejan los bosques naturales públicos -en muchos casos reconstituidos y naturalizados- de la mayoría de los países desarrollados como Bélgica, Francia, Alemania, países nórdicos, EE. UU., Reino Unido, etc., muchas veces desde hace siglos. Y el aceptable o buen estado productivo de los bosques de esos países, demuestra su buen funcionamiento y que la convivencia entre producción y conservación, aunque parece difícil, es posible. El sector privado, cuando explota bosques públicos, se libera de todos los costos y problemas que acarrea ser responsable del manejo. Por ejemplo, disponer de profesionales especializados en manejo, realizar gastos de guardianía para evitar invasiones, tener costosas certificaciones o soportar las supervisiones periódicas y multas por parte de reparticiones del gobierno. Tan solo, cuándo entra al bosque para retirar la madera que ha comprado, debe cumplir las exigencias propias de la extracción -por ejemplo, extracción de bajo impacto- establecidas en la licitación o concurso respectivo (Dourojeanni 2018c).

\section{Es preciso dar un altísimo valor a la madera de los bosques naturales}

En una nueva política forestal, la madera de los árboles nativos tropicales debe ser tratada como un producto precioso y su "precio" comenzado con el valor del canon forestal, debe ser elevado. No solamente por su belleza, raridad y diversidad, sino porque en general es el resultado de siglos de crecimiento lento, totalmente orgánico y natural. Cedro y caoba, entre tantas otras especies que tienen una gran demanda internacional, por lo cual generan hasta operaciones sofisticadas de contrabando y de corrupción, son apenas algunas del más de un millar de especies de maderas valiosísimas y hasta ahora desperdiciadas (Dancé 2006). Gran parte de las maderas "baratas", quemadas durante el desbosque o usadas para cajonería, muebles populares o encofrados en la construcción civil, si fueran tratadas, pueden transformarse en maderas preciosas para su uso en mueblería fina o para otros usos especiales.

No importa si se las exporta como productos acabados o semi-procesados o simplemente como trozas o tablones. Lo importante es que, desde el momento de su cosecha, se las trate como se merecen. Es decir, como si fueran la mejor uva para el vino más sofisticado o el tabaco de alta calidad que dará lugar a los más finos cigarros (Dourojeanni 2018c). La madera amazónica, cuando escapa al desprecio, desperdicio y maltrato que se le da en la región, se transforma en muchos de los productos más apreciados, caros y valiosos del mundo (FAO 2015). Se debe producirla con esmero y cobrar el alto precio que realmente tiene, para abastecer la gran demanda de madera certificada crecientemente insatisfecha. Apenas se logre hacer y promover la decisión de conducir la explotación maderera a ese escalón de calidad, se puede rentabilizar el manejo forestal productivo sin perjuicio para los servicios ambientales. Pero eso requiere incluir medidas ad hoc en la política forestal.

\section{La política forestal debe fijar prioridades y escalas}

Factores limitantes como el dominio de la informalidad y la corrupción están estrecha- 
mente ligadas a la ineficiencia del Estado, llegando a ponerse por encima de la legislación existente. Esta situación es en gran parte ocasionada por la vastedad y complejidad del patrimonio forestal peruano. Por eso, la política debe incluir la concentración de los esfuerzos del sector público forestal para imponerla en los aspectos prioritarios, especialmente donde tiene capacidad de hacerlo realmente, en espacios que pueden ser atendidos con los medios disponibles, evitando la enorme dilución actual. En la actualidad, todos los elementos de la política forestal pretenden ser atendidos por igual urgencia en todo el territorio nacional, sin definición de zonas prioritarias ni de etapas progresivas en que, logrado el buen control de un aspecto y área, se amplía la acción a otros temas y a otras áreas.

Así, en lugar de pretender atender $9.4 \mathrm{mi}-$ llones de hectáreas que están actualmente concedidas a cerca de 2000 empresas, sin mencionar otras modalidades de extracción, como 3 a 4000 permisos y autorizaciones (SERFOR 2017b), la nueva política debe limitar la superficie bajo manejo productivo, indispensable para mantener una industria forestal basada en los nuevos principios, debiendo pasar gradualmente a trabajar con madera proveniente de plantaciones o de vegetación secundaria manejada. Eso puede ser una fracción de lo que actualmente el gobierno se esfuerza estérilmente en controlar. Lo que quede en reserva, debe ser manejado para servicios ambientales por los que el Estado debe recibir un pago a través de los mecanismos asociados a los acuerdos sobre cambio climático.

\section{Manejo forestal en tierras indígenas y comu- nidades campesinas}

El Estado ha descuidado mucho el tema de la regularización de las tierras para los nativos amazónicos. A pesar de eso, habida cuenta del activismo político indígena, se puede partir del supuesto que los indígenas en la práctica ya tienen o tendrán control sobre la totalidad de sus tierras, que sumarían 32 millones de hectáreas, de las que 22.6 millones ya habrían sido reconocidas y tituladas (RAISG 2019), lo que es una extensión apreciable para una población de unas 350,000 personas. No toda esa tierra tiene vocación para la producción forestal pero no deja de ser significativa. Más importante aún es saber que la explotación forestal es una de las pocas y mejores opciones de desarrollo económico que ellas disponen. Dos ventajas importantes existen para el manejo forestal de esos bosques: los indígenas son dueños de esas tierras y por lo tanto las defienden contra invasores y explotadores de todo tipo y; asimismo, tienen todo interés en manejar sustentablemente el bosque, ya que ese es su patrimonio de hoy y siempre. Esas condiciones no se dan, por ejemplo, en las concesiones. En éstas, las invasiones son bienvenidas cuando acontecen después de que fue extraída la madera liberando a los concesionarios de la obligación del manejo.

La política forestal debe, pues, necesariamente dedicar un capítulo a diseñar una estrategia prioritaria para que los indígenas puedan manejar sosteniblemente sus recursos forestales, lo que implica un fuerte componente de asistencia técnica, económica, organizativa y, especialmente, financiera. Esto es urgente pues, como es bien conocido, gran parte de la extracción en esas comunidades, que es significativa a nivel nacional, es ilegal y practicada por madereros inescrupulosos o, por falta de opciones, por los propios nativos (Malleux 2010).

\section{La importancia de la forestación y la refor- estación}

Es curioso constatar que la política forestal vigente no cita el tema de la forestación y reforestación y que, pese a su evidente transcendencia, no establece ningún lineamiento político que fomente la silvicultura. La ley, como está demostrado (Dourojeanni 2013a) tampoco toca el asunto seriamente, al no brindar ningún incentivo concreto. Sin embargo, el gobierno pretende que existe más de un millón de hectáreas reforestadas, lo que es poco probable (SERFOR 2017b). Aun así, no le dedica una línea siquiera en la política nacional. 
Los bosques naturales son esenciales para mantener servicios ambientales, pero los bosques cultivados son o deben ser la base de la producción forestal industrial. Pinos y eucaliptos no se siembran, en general, para mejorar el ambiente, pero si para producir madera o resinas que alimentan industrias mecánicas o químicas. No hay gran diferencia entre plantar eucaliptos y plantar cacao, café o palma aceitera (Dourojeanni 2018c). Pero la política también debe desarrollar incentivos para la reforestación con otras especies nativas amazónicas de mayor valor maderable, como ya viene ocurriendo, a pesar de la desidia gubernamental.

El tema de la silvicultura es perfectamente coherente con las responsabilidades de cualquier ministerio de agricultura y no por eso dejaría de pertenecer al sector forestal. La forestación y reforestación merecen tener en la administración pública una institución propia y especializada, con rango elevado, similar al que se otorga a la agricultura o a la ganadería, a las que es en todo comparable. Potencialmente, la silvicultura tiene tantas o más posibilidades de expansión que esas dos actividades juntas, especialmente ocupando las tierras deforestadas abandonadas o subutilizadas y, para promoverla, existen numerosas propuestas que nunca han sido realmente implantadas (Guariguata et al. 2017). Para desarrollar la reforestación en gran escala también se necesita mucha dedicación para la creación de una estrategia de incentivos económicos propia, como se ha hecho para lanzar los programas silvícolas de Chile y Brasil, cuyos PBI forestales son muy importantes gracias a la reforestación y no a la explotación de sus bosques naturales.

Existe un área gris entre la reforestación para producción y la reforestación para restauración de ecosistemas degradados. En la primera, el objetivo económico directo -producción de madera- es subsidiario al objetivo de conservar el suelo o de garantizar el abastecimiento de agua a centros urbanos o áreas agrícolas. En esos casos, es decir en los que están rela- cionados al manejo de cuencas hidrográficas, la coordinación intersectorial debe resolver lo que se hace en cada situación (Dourojeanni 2018c).

La política forestal también debe dedicar un espacio a discutir como promover el manejo de los bosques secundarios que, en general, ocupan tierras que han sido de uso agropecuario pero que, como está demostrado, pueden contribuir mucho a la economía y a brindar empleos rurales, inclusive para los madereros informales que sean desplazados de la tala selectiva. Los bosques secundarios intensivamente manejados para la producción de madera deben ser considerados como una modalidad de silvicultura.

\section{Enfrentar la informalidad}

Un tema central que debe ser considerado en la política es el diseño de alternativas a la informalidad forestal representada por los miles de madereros pobres que salen en su mayoría de las comunidades ribereñas o de los barrios miserables de las ciudades amazónicas. Ellos son la punta de lanza del descremado en bosques de comunidades nativas, de áreas naturales protegidas o de cualquier lugar. Esos madereros sin opciones proveen materia prima barata pero ilegal, alimentando la cadena productiva corrupta que culmina en las empresas $y$ en el comercio maderero. Indirectamente, son una de las causas de la falta de manejo en las concesiones y fuera de ellas. Las alternativas de solución a esta problemática pueden incluir agruparlos en cooperativas, para lo que necesitan asesoría técnica y créditos o, dificultarles tanto el ser informales para que se vean obligados a trabajar en concesiones formales o a cambiar de actividad, entrado a actividades de reforestación o en la industria. Con estímulos adecuados es seguro que aceptarían ser formalizados.

\section{Transformar las promesas del pago por ser- vicios ambientales en realidad}

La política forestal debe abordar frontalmente el tema del pago por los servicios ambientales a aquellos que los producen, conser- 
vando o manejando bien el recurso forestal, desarrollando opciones para que se torne realidad (MINAM 2014). Como bien se sabe, el problema es que esos servicios para los peruanos y para la humanidad, a pesar de las interminables discusiones internas e internacionales, no han culminado en pagar efectivamente a los que cuidan del bosque. Ocurre que, a pesar de los esfuerzos de la corriente conocida como economía ecológica, los aportes de los servicios ambientales no han penetrado en las mediciones y cálculos de la economía convencional. Es decir, que las promesas de retribución concreta, en dólares contantes y sonantes, por conservar bosques para frenar el cambio climático o para proveer agua limpia y regular a las ciudades, no se han materializado (Dourojeanni 2018c). Este es un tema, como tantos otros en la política forestal, que debe ser acordado con otros sectores. Buenos avances han sido la creación del Programa Nacional de Bosques del MINAM y la reciente aprobación de la Ley No 30754, Ley Marco sobre Cambio Climático, aunque esta no exhibe muchas herramientas prácticas para ser aplicada.

\section{La cuestión de los bosques de protección o protectores}

Los estudios disponibles sobre la capacidad de uso mayor de los suelos del Perú son antiguos e indican, a grosso modo, que el $25 \%$ de los suelos de la Selva deberían ser conservados o protegidos (Muro 1959, Zamora 1971). Una nueva zonificación está en curso (SERFOR 2016). Aunque la cifra disponible es discutible, es evidente que la mayor parte de los bosques naturales de la Selva Alta y muchos de los que están en Costa y Sierra, en los flancos de las cordilleras, no deben ser eliminados pues se encuentran en pendientes muy fuertes, altamente erosionables y porque captan y acumulan agua. Mantenerlos no solo permite evitar desastres naturales que afectan vidas e infraestructuras, sino que garantiza el abastecimiento de agua de calidad para las ciudades y la agricultura de los valles, mantiene estable el caudal y el cauce de los ríos y alberga gran parte de la biodiversidad y del potencial turístico del país (McClain y Llerena 1998).
Pero los bosques que técnicamente son de vocación protectora o de protección -el término incluye toda la vegetación natural- no se restringen a los que cubren las fuertes pendientes andinas. En realidad, la función protectora del bosque y de la vegetación natural se extiende asimismo a las riberas de ríos, lagos y lagunas; a las nacientes de agua, al tope de las montañas, a la que cubre y fija las dunas, etc. Sin la vegetación protectora en la borda de los ríos estos reciben muchos sedimentos, salen del cauce y destruyen cultivos y viviendas, la calidad del agua que conducen se deteriora y, por ejemplo, en el caso de las dunas, éstas avanzan sobre los pueblos y la agricultura (Dourojeanni 2017).

Es verdad que parte de los bosques con vocación protectora ocurren, en mayor o menor proporción, dentro de la mayoría de las áreas naturales protegidas, tales como parques, santuarios y reservas nacionales o comunales y en algunas otras áreas naturales protegidas regionales o particulares. Quizás cubran hasta un $20 \%$ de lo que debería ser protegido en la Selva bajo el criterio técnico de bosques de protección (Dourojeanni 2018b). El resto, o sea la mayor parte de esos bosques, está dentro de tierras públicas no formalmente otorgadas y en gran parte dentro de tierras de comunidades campesinas y nativas y, obviamente, también en propiedades privadas, grandes o pequeñas. Aunque existen extensas áreas de bosques que pueden identificarse como zonas de protección o declararse oficialmente como "bosques de protección" (SERNANP) o "bosques protectores" (SERFOR), lo cierto es que los suelos que técnicamente requieren de protección están entreverados con otros y, a una escala mayor, superpuestos con otros, tanto en el paisaje como en propiedades agropecuarias, inclusive muy pequeñas. También están superpuestos y entreverados con suelos y bosques de aptitud para la producción forestal. Es decir que esos bosques están en todas partes, mezclados con otros tipos. Por ese motivo, es simplemente imposible cuidar esos bosques en forma individualizada, por lo que, en casi todos los países del mundo, ese tipo de vegetación protectora es protegido por el simple efecto de una ley. 
Las declaraciones específicas son excepcionalmente usadas para grandes espacios $y$, en ese caso, se les reconoce como una categoría de área protegida, con otro nombre.

Prueba evidente de la confusión vigente entre el uso técnico y el uso legal de las denominaciones bosque de protección o protector es que, a pesar de la importancia de cuidar esos bosques, los bosques oficiales de protección -que requieren de un complejo ritual individual de creación (SERNANP) - y los protectores, que también se crean individualmente (SERFOR) apenas abarcan 570,000 ha, es decir poco más del $0.8 \%$ de lo que realmente debería estar muy bien cuidado en la Selva y quizá apenas el $0.4 \%$ de lo que debería estar protegido en todo el territorio nacional.

Decidir cuál es el pendiente límite (en general por encima de $45^{\circ}$ ) o el ancho del bosque ribereño a ser protegido (que depende del caudal) es un tema técnico, variable con una serie de situaciones, que debe ser reflejado en la ley. Pero, las normas deben ser claras y muy simples de aplicar y verificar. La responsabilidad de proteger estos espacios debe ser de cada ciudadano y de cada propietario, y debe ser realizada por las autoridades agrarias, forestales y ambientales, asimismo por el Ministerio Público y la policía que cuida del ambiente, pues no proteger estos bosques implica perjuicios graves para toda la sociedad. La falta de respeto a esa legislación debe implicar la imposibilidad de acceso a préstamos agrarios, la compra y venta o alquiler de propiedades rurales, además de multas y la obligación de recuperar o compensar los bosques protectores que fueran destruidos.

\section{Otras consideraciones por desarrollar en la política forestal}

Lo anterior es apenas la mención de temas centrales, cruciales, pero hay muchos más que deben ser discutidos en una política forestal y en la ley que se desprenda de ella. Como por ejemplo el tema de la fauna silvestre que, en opinión de este autor, no debe ser incluida en la política forestal. La conservación y manejo de la fauna silvestre es un tema obviamente asociado al bosque, pero con tantas peculiaridades y requerimientos profesionales diferentes, que no tiene sentido alguno colocar su gestión dentro de una administración forestal. No debe olvidarse que gran parte de la fauna peruana, inclusive algunos de sus representantes más famosos y valiosos, como vicuña y aves guaneras, no habitan ecosistemas forestales. La fauna silvestre merece tener su propia gestión, como en la mayor parte de los países del mundo.

Un asunto extremamente importante es discutir la validación actual de la llamada clasificación de tierras por capacidad de uso mayor. Ésta, en opinión del autor, ya cumplió su rol y es tiempo de considerar su sustitución por reglas más simples y fácilmente aplicables. Las tierras tienen, obviamente, una aptitud natural para cada tipo de uso. Aprovechar esa aptitud natural, en principio, ahorra esfuerzo y costos para usarlas y evita que sean degradadas. Pero eso vale para grandes unidades geográficas, y no para la ocupación de la tierra por la agricultura formal e informal. En efecto, la Amazonía, especialmente en las tierras bajas, la superposición entre capacidades de uso agropecuario y forestal es muy elevada y puede variar en pocos metros, debiéndose aplicar un promedio que no siempre es adecuado. Además, detectar eso requiere estudios detallados que están fuera de la viabilidad económica. Sin embargo, la principal razón para no seguir usando ese método es que los avances tecnológicos agropecuarios han eliminado la calidad del suelo como factor limitante, siendo este ahora determinado por la economía, asociada a la disponibilidad de infraestructura de transporte (EMBRAPA 2006). Lo que no se podía cultivar rentablemente veinte o treinta años atrás, ahora es perfectamente posible.

Finalmente, si se acepta que la principal función del bosque natural remanente es proveer servicios ambientales y no madera, resulta obvio que las agencias encargadas de su gestión deben depender del Ministerio del Ambiente. En la actualidad, por lo contrario, esa responsabilidad está dividida y tironeada entre tres sectores (Agricultura, Ambiente y la Presidencia del Consejo de Ministros) además de las regiones. Eso ocurre a pesar de que ninguna 
de las agencias o reparticiones de ese conjunto tiene capacidad institucional para ser efectivas. Habida cuenta que las tres partes nacionales son esencialmente técnico-normativas o, por lo contrario, supervisoras o controladoras, no existe motivo alguno para no unirlas en una sola, más aún si se acaba con el sistema de concesiones, que es el foco de la corrupción en el sector (Dourojeanni 2018a, 2018c). En términos de ejecución, la única responsabilidad que en gran medida es nacional es la investigación y su extensión o difusión. La ejecución de la política forestal debe recaer esencialmente en las administraciones forestales regionales, con apoyo nacional.

\section{Conclusión}

Ningún pronóstico sobre el futuro de los bosques naturales, especialmente los tropicales, es animador. Desde hace décadas muchos investigadores consideraron que el manejo sostenible del bosque tropical es apenas una utopía (Gómez-Pompa et al. 1972). Para Blaser y Gregersen (2013), entre otros, no hay posibilidad de evitar que los bosques primarios tropicales se reduzcan a una mínima expresión o desparezcan en el transcurso de este y de los próximos dos siglos, por la acción combinada de la expansión agropecuaria -incluyendo como parte de ésta a la reforestación con especies nativas u exóticas- y del cambio climático. Auguran que continuarán existiendo bosques, quizá con mayor extensión que en la actualidad, pero que serán muy diferentes, es decir que casi todos serán provenientes de la reforestación, por lo tanto artificiales o cultivados.

Es posible que los pesimistas tengan razón, como las tendencias parecen probar, pero ese no es motivo para diseñar una política forestal que contribuya a aumentar la velocidad del proceso de deforestación y desperdicio de los bosques naturales. A este punto, una vez más, se debe tomar una decisión: se acata lo que parece inexorable y, en ese caso, salvo defender las áreas naturales protegidas, se acepta que todo el resto de los bosques pueden ser explotado sin cuidado, ya que se sabe que más temprano que tarde será deforestado y usado para fines agropecuarios o; por lo contrario, se adopta una política de defensa del bosque natural basada en la procura de un verdadero manejo "sostenible", del que tanto se habla y por el cual se hace tan poco. Lo que no es lógico es continuar con la hipocresía de hace creer que la actual política forestal sirve para mantener el patrimonio forestal cuando todas las evidencias indican que contribuye a su destrucción.

\section{Bibliografía}

Akishin, V. 2014. Forest planning as the most important aspect of sustainable forest management. ERSA conference papers ersa14p569, European Regional Science Association. Consultado 20 abr 2019. Disponible en https:// ideas.repec.org/p/wiw/wiwrsa/ersa14p569. html

Arce, JM; Dourojeanni, M. 1990. Análisis crítico sobre el cumplimiento de la Ley Forestal y de Fauna Silvestre. Revista Forestal del Perú 17(2):03-20.

Blaser, J; Gregersen, H. 2013. Forests in the next 300 years. Unasylva 240(64):61-73. Consultado 20 abr 2019. Disponible en https://www.eda. admin.ch/dam/deza/en/documents/themen/ klimawandel/223023-forests-next-300-years EN.pdf

Brack, A. 2010. Para el 2021 esperamos reducir a cero la deforestación en la Amazonía y costa norte. Actualidad Ambiental, SPDA. Lima. Consultado 20 abr 2019. Disponible en http:// www.actualidadambiental.pe/?p=7239

Brown, S; Zarin, D. 2013. What Does Zero Deforestation Mean? Environmental Science. Science 342:805-807. Consultado 20 abr 2019. Disponible en https://www.researchgate.net/ publication/258529149 What Does Zero Deforestation Mean

Carton, C; Chavez, A. 2018. Porcón. Medio Siglo de Forestación en los Andes de Cajamarca. Virrey, Lima. Consultado 20 abr 2019. Disponible en https://rcrperu.com/porcon- 
medio-siglo-de-forestacion-en-los-andes-decajamarcal

Cerdán, C. 2007. La tala Ilegal de caoba (Swietenia macrophylla) en la Amazonía peruana y su comercialización al mercado exterior. Asociación Interétnica de Desarrollo de la Selva Peruana (AIDSEP), Lima. Consultado $20 \mathrm{abr}$ 2019. Disponible en https://launderingmachine.files.wordpress.com/2012/04/tala_ilegal de caoba en peru aidesep.pdf

Che Piu, H. 2018. Legislación forestal peruana ¿Mucha norma jurídica, poca ley y derecho? El dilema del sector forestal peruano. Consultado 22 abr 2019. Disponible en http://dialogosdelareladefa.blogspot.com/2018/04/legislacion-forestal-peruana-1-che-piu.html

Chirif, A (ed.) Deforestación en tiempos de cambio climático. 2018. IWGIA, Lima. Consultado 20 abr 2019. Disponible en https:// searchworks.stanford.edu/view/12803886

Dancé, J. 2006. Introducción de Especies Líderes en los Mercados de Exportación. Sexto Informe Bimensual. Programa de Desarrollo de Políticas de Comercio Exterior 1442/OC-PE. Dirección General de Desarrollo de Comercio Exterior. Lima.

Defensoría del Pueblo. 2010. La Política Forestal y la Amazonía Peruana: Avances y obstáculos en el camino hacia la sostenibilidad. Serie Informes Defensoriales Informe $\mathrm{N}^{\circ}$ 151. Consultado 15 abr 2019. Disponible en https://www.defensoria.gob.pe/wp-content/ uploads/2018/05/informe-151.pdf

Dourojeanni, M. 1990. Amazonía ¿Qué hacer? Iquitos, Perú Centro de Estudios Teológicos de la Amazonía (CETA). Consultado 20 abr 2019. Disponible en www.gbv.de/dms/sub-hamburg/042846226.pdf

Dourojeanni, M. 1999. The future of Latin American's natural forests. In Keipi, K (ed.). Forest Resource Policy in Latin America. Inter-American Development Bank/John Hopkins Univ. Press, Washington, D.C. Consultado 3 abr 2019. Disponible en https:// pt.scribd.com/document/335796333/The-future-of-the-Latin-American-tropical-forests
Dourojeanni, M. 2009. Crónica Forestal del Perú. Universidad Nacional Agraria La Molina, Editorial San Marcos, Lima. Consultado 15 abr 2019. Disponible en https://www.academia. edu/14408042/Crónica Forestal del Perú

Dourojeanni, M. 2010. ¿Una nueva ley forestal en el Perú? Xilema 23(1): 5-10. Consultado 20 abr 2019. Disponible en http://revistas.lamolina.edu.pe/index.php/xiu/article/ view/679/662

Dourojeanni, M. 2011. Amazonia Probable y deseable. Ensayo sobre el Presente y Futuro de la Amazonía Peruana. Univ. Inca Garcilaso de la Vega. Textos Universitarios/Ecología. Lima.

Dourojeanni, M. 2013a. La nueva legislación forestal en el Brasil y en el Perú. Xilema 26:5-9. Consultado 18 abr 2019. Disponible en http:// revistas.lamolina.edu.pe/index.php/xiu/article/download/608/591

Dourojeanni, M. 2013b. Análisis crítico de la ley forestal peruana. Agenda Ambiental 2(4): 20-32. Consultado 20 abr 2019. Disponible en https://www.academia.edu/5669131/Analisis critico de la ley forestal peruana

Dourojeanni, M. 2013c. Misterios de la deforestación en el Perú. Actualidad Ambiental. SPDA. Lima. Consultado 13 abr 2019. Disponible en https://www.actualidadambiental. pe/?p=19955

Dourojeanni, M. 2017. La importancia de los bosques para enfrentar inundaciones y aluviones. Actualidad Ambiental, SPDA. Lima. Consultado 20 abr 2019. Disponible en http:// www.actualidadambiental.pe/?p=44523

Dourojeanni, M. 2018a. Comentarios sobre decisiones recientes relativas a gestión forestal y deforestación. Actualidad Ambiental, SPDA. Lima. Consultado 20 abr 2019. Disponible en http://www.actualidadambiental.pe/?p=53774/

Dourojeanni, M. 2018b. Bosques protegidos y protectores en el Perú, Actualidad Ambiental, SPDA, Lima. Consultado 18 abr 2019. Disponible en http://www.actualidadambiental. $\mathrm{pe} / \mathrm{p}=50188$ 
Dourojeanni, M. 2018c. Ocho errores capitales de la gestión forestal en el Perú. Actualidad Ambiental, SPDA. Lima. Consultado 19 abr 2019. Disponible en http://www.actualidadambiental.pe/?p=52065

Dourojeanni, M; Ráez, E; Valle-Riestra, E. 2016. Ambiente y Recursos naturales en el Perú. Quinquenio 2011-2016. DAR, Lima. Consultado 20 abr 2019. Disponible en https:// www.dar.org.pe/archivos/publicacion/180 quinquenio.pdf

Egúsquiza, CP. 2012. Una visión crítica del pago por servicios ambientales. Actualidad Ambiental, SPDA. Lima. Consultado 15 abr 2019. Disponible en http://www.actualidadambiental.pe/wp-content/uploads/2012/10/ Una-visi\%C3\%B3n-cr\%C3\%ADtica-del-Pago-por-Servicios-Ambientales.pdf

Espinoza, R; Feather, C. 2011. La realidad de REDD+ en Perú: entre el dicho y el hecho. Forest Peoples Program/Aidesep/Fenamad, Lima. Consultado 20 abr 2019. Disponible en http://www.forestpeoples.org/sites/fpp/files/ publication/2011/11/la-realidad-de-redd-enperu-entre-el-dicho-y-el-hecho-para-el-sitioweb 0.pdf

EIA (Environmental Investigation Agency). 2010. The LaunderIng MachIne. How fraud and corruption in Peru's concession system are destroying the future of its forests. EIA Global. Washington, DC. Consultado 26 abr 2019. Disponible en https://content.eia-global.org/ posts/documents/000/000/501/original/The Laundering Machine ENG.pdf?1475785276

EMBRAPA (Empresa Brasileira de Pesquisa Agropecuária). 2006. Agricultura tropical: o Brasil produzindo o futuro. Empresa Brasileira de Pesquisa Agropécuaria. Brasília. Consultado 26 abr 2019. Disponible en https://ainfo.cnptia. embrapa.br/digital/bitstream/item/60916/1/ Agricultura-tropical.pdf

Fagan, C; Shoobridge, D. 2005. Una Investigación de la Extracción Ilegal de Madera en el Parque Nacional Alto Purús y Alrededores. ParksWatch, Lima. Consultado 19 abr 2019. Disponible en https://www.parkswatch.org/ spec reports/logging apnp spa.pdf
FAO (Food and Agriculture Organization). 2010a. ¿Qué es una política forestal nacional? FAO, Roma. Consultado 19 abr 2019. Disponible en http://www.fao.org/3/i1679s/ i1679s03.pdf

FAO (Food and Agriculture Organization). 2010b. Elaboración de una política forestal eficaz. FAO, Roma. Consultado 20 abr 2019. Disponible en http://www.fao.org/3/am007s/ am007s00.pdf

FAO (Food and Agriculture Organization). 2015. Assessment of main marketing factors used in accessing markets for tropical timber products. FAO, Roma. Consultado $20 \mathrm{abr}$ 2019. Disponible en http://www.fao.org/3/ y2199e/y2199e08.htm

Finer, M; Jenkins, CN; Blue, MA; Pine, J. 2014. Logging Concessions Enable Illegal Logging Crisis in the Peruvian Amazon. Scientific Reports. Consultado 20 abr 2019. Disponible en https://www.researchgate.net/publication/261757367 Logging Concessions Enable Illegal Logging Crisis in the Peruvian Amazon

Foley, JA; Asner, GP; Costa, MH; Coe, MT; De Fries, R; Gibbs, HK; Howard, EA; Olson, S; Patz, J; Ramankutty, N; Snyder, P. 2007. Amazonia revealed: forest degradation and loss of ecosystem goods and services in the Amazon Basin. Frontiers in Ecology and the Environment 5: 25-32. Consultado 17 abr 2019. Disponible en https://experts.umn.edu/en/ publications/amazonia-revealed-forest-degradation-and-loss-of-ecosystem-goods-

Galarza, E; La Serna, K. 2005. ¿Son sostenibles las concesiones forestales en el Perú? CIES. Economía y Sociedad 56. Consultado 17 abr 2019. Disponible en. http://infobosques.com/portal/wp-content/uploads/2016/05/05-galarza.pdf

Glave, M; Pizarro, R (eds.). 2002. Valoración económica de la diversidad biológica y servicios ambientales en el Perú. INRENA. Consultado 17 abr 2019. Lima. Disponible en http:// www.grade.org.pe/publicaciones/540-valoracion-economica-de-la-diversidad-biologica-y-servicios-ambientales-en-el-peru/ 
Global Witness. 2019. El justiciero forestal: Por qué se debe devolver la independencia a OSINFOR y expandir sus poderes Informe Global Witnes. Consultado 20 abr 2019. Disponible en https://www.globalwitness.org/documents/19591/El justiciero forestal.pdf

Gómez-Pompa, A; Vásquez-Yáñez, C; Guevara, C. 1972. The tropical rain forests: A non-renewable resources. Science 177(4051):762-764. Consultado 13 abr 2019. Disponible en https:// www.ncbi.nlm.nih.gov/pubmed/17840118

de Groot, R; Brander, L; van der Ploeg, S; Costanza, R; Bernard, F; Braat, L; Christie, M; Crossman, N; Ghermandi, A; Hein, L; Hussain, S; Kumar, P; McVittie, A; Portela, R; Rodriguez, LC; ten Brink, P; van Beukering, P. 2012. Global estimates of the value of ecosystems and their services in monetary units. Ecosystem Services 1(1):50-61. Consultado 12 abr 2019. Disponible en https://www.sciencedirect.com/ science/article/pii/S2212041612000101

Guariguata, M; Arce, J; Ammour, T; Capella, JL. 2017. Las plantaciones forestales en Perú. Reflexiones, estatus actual y perspectivas a futuro. Documento Ocasional 169. Bogor, Indonesia: CIFOR. Consultado 21 abr 2019. Disponible en https://www.cifor.org/library/6461/

INEI (Instituto Nacional de Estadística e Informática). 2017. Indicadores Económicos y Sociales (en línea, sitio web). Consultado 20 abr 2019. Disponible en https://www.inei. gob.pe/biblioteca-virtual/boletines/indicadores-economicos-y-sociales/1/

INEI (Instituto Nacional de Estadística e Informática). 2018. Eventos Naturales, Antrópicos y Desastres (en línea, sitio web). Consultado 21 abr 2019. Disponible en https://www.inei. gob.pe/estadisticas/indice-tematico/d-eventos-naturales-antropicos-y-desastres-10304/

León, F. 2007. El aporte de las áreas naturales protegidas a la economía nacional. Instituto Nacional de los recursos Naturales, Lima. Consultado 16 abr 2019. Disponible en http:// infobosques.com/portal/wp-content/uploads/2016/04/aporte_areas_naturales_protegidas fernando leon.pdf
León, F; Rodriguez, A; Drumm, A; Murrugarra, F; Lindberg, K; Gonzales, C. 2009. Valoración económica del turismo en el sistema nacional de áreas naturales protegidas por el Estado: un estudio de caso en cuatro áreas naturales protegidas del Perú. Ministerio del Ambiente. The Nature Conservancy. Lima.

Llerena, C; Yalle, S. 2014. Los servicios ecosistémicos en el Perú. Xilema 27:62-75. Consultado 21 abr 2019. Disponible en http://revistas.lamolina.edu.pe/index.php/xiu/article/ view/177

MAAP (Monitoring of the Andean Amazon Project). 2017. Synthesis \#2: Patterns and Drivers of Deforestation in the Peruvian Amazon. Consultado 22 abr 2019. Disponible en http:// maaproject.org/2017/maap-synthesis $2 /$

MAAP (Monitoring of the Andean Amazon Project). 2018a. Síntesis \#3: Deforestación en la Amazonía andina (tendencias, hotspots y drivers). Consultado 22 abr 2019. Disponible en https://maaproject.org/2018/sintesis3/

MAAP (Monitoring of the Andean Amazon Project). 2018b. MAAP \#92: Nuevas amenazas de deforestación en la Amazonía peruana (parte 2: expansión agrícola). Setiembre 10, 2018. Consultado 22 abr 2019. Disponible en https:// maaproject.org/2018/nuevas-amenazas-2/

Makarieva, AM; Gorshkov, VG; Sheil, D; Nobre, AD: Bunyard, P; Li, B-L. 2014. Why does air passage over forest yield more rain? Examining the coupling between rainfall, pressure, and atmospheric moisture content. Journal of Hydrometeorology 15:411-426.

Malleux, R. 2010. Tenencia de bosques en el Perú. FAO, Lima. 27 p. Consultado 20 abr 2019. Disponible en www.fao.org/forestry/ download/17195-0fb06a577bfa399fcadef97baaf6a835d.pdf

Marengo, JÁ; Tomasella, J; Soares, WR; Alves, LM; Nobre, CA. 2011. Extreme climatic events in the Amazon basin. Theoretical and Applied Climatology 107:73-85.

McClain, ME; Llerena, C. 1998. El manejo de cuencas en la selva: de los Andes a la Amazonia. Agua y Riego 11:11-13. 
McClain, ME: Victoria, RL; Richey, JE (eds.). 2001. Biochemistry of the Amazon basin. Oxford, U.K.: Oxford University Press. Consultado 23 abr 2019. Disponible en https://global. oup.com/academic/product/the-biogeochemistry-of-the-amazon-basin-9780195114317?cc=br\&lang=en \&

MINAM (Ministerio del Ambiente). 2014. Amazonia y cambio climático: Perspectivas, oportunidades y amenazas. Ministerio del Ambiente, Lima. Consultado 20 abr 2019. Disponible en https://centroderecursos.cultura. pe/es/registrobibliografico/amazon\%C3\%ADa-y-cambio-clim\%C3\%A1tico-perspectivas-oportunidades-y-amenazas- 0

MINAM (Ministerio del Ambiente). 2015. Mapa de susceptibilidad física del Perú. Zonas propensas a inundaciones y deslizamientos en la costa y sierra frente a la ocurrencia de eventos hidrometeorológicos extremos. Documento de Trabajo 2015. Ministerio del Ambiente, Lima. Consultado 19 abr 2019. Disponible en http://www.minam.gob.pe/wp-content/uploads/2017/01/mapa-de-susceptibilidad-fisica. pdf

Muro, J. 1959. Consideraciones sobre los suelos agrícolas de la región de tropical del Perú. Bases Fisiológicas de la Producción Agrícola. Lima, IICA, Zona Andina.

Myers, N. 1984. The Primary Source: Tropical Forests and Our Future. Synergism.

Nobre, C; Sampaio, G; Borma, LS; Castilla-Rubio, JC; Silva, JS; Cardoso, M. 2016. Land-use and climate change risks in the Amazon and the need of a novel sustainable development paradigm. PNAS 113(39):10759-10768. Consultado 23 abr 2019. Disponible en https:// www.pnas.org/content/113/39/10759

Pautrat, L; Lucich, I. 2006. Análisis preliminar sobre gobernabilidad y cumplimiento de la legislación del Sector Forestal en el Perú. World Bank Group. Washington, DC. Consultado 20 abr 2019. Disponible en http://www.keneamazon.net/Documents/Publications/Virtual-Library/Marco-Normativo/66.pdf
Pautrat, L; Torres, P; Samaniego, C. 2010. Aportes a la propuesta de proyecto de ley forestal y de fauna silvestre. Sociedad Peruana de Ecodesarrollo. Lima. Consultado $20 \mathrm{abr}$ 2019. Disponible en https://pt.scribd.com/ document/167148797/Aportes-a-La-Legislacion-Forestal-01

Prance, G. T.; Lovejoy, T, E. (eds.) 1984. Key Environments. Amazonia. Pergamon. Oxford.

Putz, FE; Romero, C. 2015. Futures of tropical production forests. CIFOR Occasional Paper No. 143. Consultado 20 abr 2019. Disponible en http://www.cifor.org/publications/pdf files/ OccPapers/OP-143.pdf

RAISG (Red Amazónica de Información Socioambiental Georreferenciada). 2019. Amazonía 2019 - Áreas Protegidas y Territorios Indígenas. Red Amazónica de Información Socioambiental Georreferenciada. Consultado 30 abr 2019. Disponible en https://www.amazoniasocioambiental.org/es/publicacion/amazonia-2019-areas-protegidas-y-territorios-indigenas/

Salati, E; Dall'Olio, A; Matsui, JRG. 1979. Recycling of water in the Amazon basin: An isotopic study. Water Resources Research 15:12501258.

Salati, E; Vose, P. 1984. Amazon basin: A system in equilibrium. Science, 225(4568):129205. Consultado 20 abr 2019. Disponible en http://www.eebweb.arizona.edu/faculty/saleska/Ecol596L/Readings/Salati.Vose84_Amazon Science.pdf

Salo, M; Puro, JP; Knuuti, K. 2013. Control and intimacy in the Amazon reality: Newspaper rethoric on forest sector reform in Peru. Land and Use Policy 35:226-236. Consultado 20 abr 2019. Disponible en https://www. sciencedirect.com/science/article/abs/pii/ $\underline{\text { S0264837713001142 }}$

SERFOR (Servicio Nacional Forestal y de Fauna Silvestre). 2013. Política Nacional Forestal y de Fauna Silvestre. Ministerio de Agricultura y Riego. Lima. 22 p. Consultado 22 abr 2019. Disponible en http://minagri.gob.pe/portal/ download/pdf/especiales/politica-nacional.pdf 
SERFOR (Servicio Nacional Forestal y de Fauna Silvestre). 2016. Guía Metodológica para la Zonificación Forestal. Ministerio de Agricultura y Riego. Lima. 42 p. Consultado 20 abr 2019. Disponible en https://www.serfor. gob.pe/wp-content/uploads/2016/12/SERFOR-Guía-Metodológica.pdf

SERFOR (Servicio Nacional Forestal y de Fauna Silvestre). 2017a. Política Nacional Forestal y de Fauna: Edición de bolsillo). Ministerio de Agricultura y Riego. Lima 78 p. Consultado 22 abr 2019. Disponible en https://www.serfor. gob.pe/wp-content/uploads/2017/06/Politica $\% 20$ Nacional $\% 20$ Forestal $\% 20 \mathrm{y} \% 20 \mathrm{de} \% 20$ Fauna\%20Silvestre\%20-\%20Edicion \%20 de\%20bolsillo.pdf

SERFOR (Servicio Nacional Forestal y de Fauna Silvestre). 2017b. Anuario Forestal 2017. Ministerio de Agricultura y Riego. Lima. 124 p. Consultado 22 abr 2019. Disponible en https://www.serfor.gob.pe/wp-content/uploads/2019/02/Anuario-Forestal-y-de-Fauna-Silvestre-2017.pdf

Sierra Y. 2018a. La ruta de la tala selectiva y su impacto en la Amazonía peruana. Mongabay Series: Global Forests, 31 octubre 2018. Consultado 18 abr 2019. Disponible en https:// es.mongabay.com/2018/10/la-ruta-de-la-tala-selectiva-y-su-impacto-en-la-amazonia-peruana/

Sierra, Y. 2018b. Moment of truth: Study reveals high percentage of illegal Peruvian timber exports. Experts fear that the lack of transparency in Peruvian timber exports will lead to the closure of international markets. Mongabay Series: Global Forests. 13 February 2018. Consultado 20 abr 2019. Disponible en https://news.mongabay.com/2018/02/moment-of-truth-study-reveals-high-percentage-of-illegal-peruvian-timber-exports/

Sierra, Y. 2019a. Madre de Dios: Seven Brazil nut concessions investigated for illegal timber extraction Mongabay Series: Global Forests. 23 February 2019. Consultado 20 abr 2019. Disponible en https://news.mongabay. com $/ 2019 / 02 / \mathrm{madre}$-de-dios-seven-bra- zil-nut-concessions-investigated-for-illegal-timber-extraction/

Sierra, Y. 2019b. Peru: Report reveals high rates of illegality in timber extraction. Mongabay Series: Global Forests. 14 February 2019. Consultado 23 abr 2019. Disponible en https://news.mongabay.com/2019/02/ peru-report-reveals-high-rates-of-illegality-in-timber-extraction/?n3wsletter\&utm_source $=$ Mongabay + Newslet ter\&utm campaign $=\mathrm{f} 42 \mathrm{f} 6 \mathrm{cfcab}$-newsletter 201902 21\&utm medium=email\&utm term $=0 \quad 940652 \mathrm{e} 1 \mathrm{f} 4-\mathrm{f} 42 \mathrm{f} 6 \mathrm{cfcab}-67233711$

Suárez, G. 2012. Elementos para la Política Forestal en el Perú. Lima. Consultado $20 \mathrm{abr}$ 2019. Disponible en http://infobosques.com/ portal/wp-content/uploads/2016/03/elementos para la politica forestal en el peru.pdf

Vilela, T; Rubio, JC; Escobedo, AJ; Bruner, A; Conner, N. 2018. El impacto económico local del turismo en áreas protegidas del Perú. Documento de Trabajo. Conservation Strategy Fund. Consultado 20 abr 2019. Disponible en https://www.conservation-strategy.org/sites/ default/files/field-file/ES Documento de trabajo Peru Tourism Multipliers Feb 20180. pdf

Zamora, C. 1971. Capacidad de uso de los suelos del Perú (3a aprox.). Oficina Nacional de Evaluacion de Recursos naturales. ONERN. Lima.

Zimmerman, B; Kormos, C. 2012. Prospects for Sustainable Logging in Tropical Forests. BioScience 62(5): 479-487. Consultado 22 abr 2019. Disponible en https://academic.oup. com/bioscience/article/62/5/479/236463 